\title{
BMJ Open Protocol for a large-scale prospective observational study with alogliptin in patients with type 2 diabetes: J-BRAND Registry
}

\author{
Nobuya Inagaki, ${ }^{1}$ Kohjiro Ueki, ${ }^{2}$ Yukio Tanizawa, ${ }^{3}$ Hirotaka Watada, ${ }^{4}$ \\ Jiro Nakamura, ${ }^{5}$ Yuichiro Yamada, ${ }^{6}$ lichiro Shimomura, ${ }^{7}$ Rimei Nishimura, ${ }^{8,9}$ \\ Tsutomu Yamazaki, ${ }^{10}$ Takashi Kadowaki ${ }^{2}$
}

To cite: Inagaki N, Ueki K, Tanizawa $Y$, et al. Protocol for a large-scale prospective observational study with alogliptin in patients with type 2 diabetes: J-BRAND Registry. BMJ Open 2014;4: e004760. doi:10.1136/ bmjopen-2013-004760

- Prepublication history for this paper is available online. To view these files please visit the journal online (http://dx.doi.org/10.1136/ bmjopen-2013-004760).

Received 27 December 2013

Revised 21 May 2014

Accepted 22 May 2014

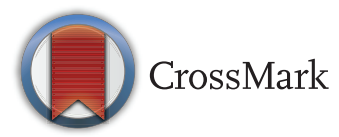

For numbered affiliations see end of article.

Correspondence to Dr Nobuya Inagaki; inagaki@metab.kuhp. kyoto-u.ac.jp

\section{ABSTRACT}

Introduction: Dipeptidyl peptidase-4 (DPP-4) inhibitors including alogliptin are categorised as a newer class of oral hypoglycaemic, antidiabetic drugs to suppress the degradation of incretin hormones ((glucagon-like peptide-1 (GLP-1) and glucosedependent insulinotropic polypeptide (GIP)) by DPP-4. We have scheduled a large-scale, multicentre, prospective, observational study (Japan-Based clinical ReseArch Network for Diabetes Registry: J-BRAND Registry) to construct an extensive database over a long-term clinical course in patients with type 2 diabetes receiving oral hypoglycaemic agents (OHAs) and to evaluate the safety and efficacy of alogliptin in Japanese population.

Methods and analysis: 20000 patients with type 2 diabetes will be registered into two groups of 10000 each: group A patients will be treated with alogliptin, while group $B$ patients will be treated with non-DPP-4 inhibitor OHA(s). Approximately 300 institutions nationwide will enrol and assign eligible patients equally to either group. Each patient's data will be collected every 6 months for a 3-year period, during which time treatment with $\mathrm{OHA}(\mathrm{s})$ may be changed or discontinued, as per package insert for each $\mathrm{OHA}$. Primary end points are safety variables to be compared between the two groups and their subgroups, with respect to hypoglycaemia, pancreatitis, skin disorders, infections and cancer. Secondary end points are efficacy variables including from-baseline changes of A1c, fasting glucose, fasting insulin and urinary albumin, which will be compared between groups/subgroups. New onset and progression of microangiopathy will also be evaluated against $\mathrm{OHA}(\mathrm{s})$. Overall, the J-BRAND Registry will evaluate the safety and efficacy of antidiabetic $\mathrm{OHA}$ (s) including alogliptin, based on a large-scale database. Ethics and dissemination: This study will be conducted with the highest respect for individual participants according to this protocol, the Declaration of Helsinki, the Ethical Guidelines for Clinical Research (Japan Ministry of Health, Labour and Welfare, 2008) and relevant laws/regulations. The present study will construct a valuable database of patients with type 2 diabetes treated with $\mathrm{OHA}(\mathrm{s})$ including alogliptin.

Trial registration number: UMIN000007976.
Strengths and limitations of this study

- This study will be conducted as a first nonrandomised, observational study to establish a large-scale database with regard to the safety and efficacy profiles of a dipeptidyl peptidase-4 (DPP-4) inhibitor in comparison to non-DPP-4 inhibitor oral hypoglycaemic agents.

- The database is expected to promote appropriate use of DPP-4 inhibitors when used alone or in combination with other antidiabetic agents.

- It will need several years for the full construct of database.

\section{INTRODUCTION}

Type 2 diabetes mellitus is a metabolic disease in which patients experience chronic hyperglycaemia and is very often associated with various complications including macrovascular as well as microvascular diseases, such as cardiovascular disease, retinopathy, nephropathy and neuropathy. As of 2011, an estimated 366 million people have been affected with diabetes globally including Japan, where more than 24 million people have been diagnosed or are suspected to have diabetes ${ }^{12}$ and the prevalence is rapidly increasing worldwide. ${ }^{1-5}$

There have been different classes of agents developed for the treatment of type 2 diabetes including insulin and oral hypoglycaemic agents (OHAs). ${ }^{3-6}$ Among those, incretinrelated drugs have been noted in recent years as a novel class of antidiabetic agents ${ }^{4-7}$ and are widely used in daily clinical practice, expanding the range of treatment options for patients with type 2 diabetes. Specifically, dipeptidyl peptidase-4 (DPP-4) inhibitors have attracted clinical attention because of the convenience of once-daily or twice-daily oral administration and the pancreatic $\beta$-cell protective effect, ${ }^{8}$ which conventional OHAs for 
type 2 diabetes do not usually provide. Additionally, DPP-4 inhibitors do not induce weight gain but may cause hypoglycaemia, though not frequently. ${ }^{9}$ As a consequence, the amount of DPP-4 inhibitors prescribed has been increasing exponentially and many patients with type 2 diabetes currently receive a DPP-4 inhibitor concomitantly with other drug classes in daily clinical practice.

In order to promote appropriate use of DPP-4 inhibitors, it is necessary to investigate the safety and efficacy of combination therapies with this drug class and various other agents. For example, hypoglycaemia is one of the issues of interest, but no such data have yet been systematically obtained in association with the use of DPP-4 inhibitors. In recent reports, the possibility of an increasing risk of pancreatitis, skin disorders, infections and cancer has been suggested in DPP-4 inhibitor-treated patients ${ }^{10-12}$; however, these events are rare, and it seems difficult to associate the drug class with such risks on the basis of non-clinical and clinical data currently available.

A considerable number of diabetes-related databases were constructed in the USA and Europe, and their stratified analyses have provided results that associate specific drugs with efficacy or safety data. ${ }^{13} 14$ These results have been reflected in clinical practice guidelines to establish standard treatment, making a contribution to the development of pharmacotherapy. However, there are no databases worldwide with regard to DPP-4 inhibitors, because this drug class has only recently been launched. This situation presents an urgent need to accumulate safety and efficacy data to support evidence-based medicine (EBM) for treatment with DPP-4 inhibitors and other OHAs. We therefore have scheduled a prospective, observational study (Japan-Based clinical ReseArch Network for Diabetes Registry: J-BRAND Registry) of actual cases with long-term experience in daily clinical practice: this type of research may be as useful for practising EBM as is an interventional, randomised study. Since DPP-4 inhibitors, particularly alogliptin (Nesina), have recently been implicated in a beneficial, antiatherogenic mechanism to reduce the risk of cardiovascular events, ${ }^{15-17}$ alogliptin was chosen as a representative DPP-4 inhibitor throughout the J-BRAND Registry study. The drug will be administered to the planned 10000 patients, as per its package insert $(25 \mathrm{mg}$ once daily, except for in patients associated with moderate-to-severe kidney malfunction, who are to receive either 6.25 or $12.5 \mathrm{mg}$ daily at physician's discretion), while OHAs other than DPP-4 inhibitors will be used in another 10000 patients for comparison (see Methods and analysis). Based on safety and efficacy information to be obtained through this research, we expect to construct a database of cases from daily clinical practice and hence to promote appropriate use of DPP-4 inhibitors when used alone or in combination with other agents.

\section{METHODS AND ANALYSIS}

The objective of this prospective study is to construct a database regarding the long-term (3-year) clinical course in patients with type 2 diabetes who receive OHAs in daily clinical practice and to evaluate the safety and efficacy of alogliptin, a novel DPP-4 inhibitor.

\section{Primary end points}

The incidence, type and severity of adverse events will be compared between group A patients initiated with alogliptin treatment and group B patients initiated with OHA treatment other than DPP-4 inhibitors at the time of registration (between-group comparison) and among subgroups defined by baseline characteristics and by concomitant medication (between-subgroup comparison). All adverse events will be included in the safety evaluation, and major safety concerns will include hypoglycaemia, pancreatitis, skin disorders, infections and cancer.

\section{Secondary end points}

Efficacy variables will be compared according to patient grouping or subgrouping as above. The efficacy variables will include the changes from baseline values (at the time of registration) in the levels of Alc (glycated haemoglobin (HbA1c); National Glycohemoglobin Standardization Program (NGSP)), fasting blood glucose, fasting insulin and urinary albumin, as well as the effect of OHA(s) on the new onset of microangiopathy (diabetic retinopathy, diabetic nephropathy, diabetic neuropathy) and its progression.

\section{Other measurements}

Laboratory tests include serum lipids such as highdensity lipoprotein cholesterol, low-density lipoprotein cholesterol and triglycerides. Standard 12-lead ECGs, weight and diastolic and systolic blood pressure in a sitting position will also be measured.

\section{Overall study design}

This is a large-scale, multicentre, prospective, observational study in which 20000 patients with type 2 diabetes will be consecutively registered by a central registration procedure. An OHA should be newly started, added to previous treatment or switched from the previous $\mathrm{OHA}(\mathrm{s})$ in patients at the time of study registration. The type of a newly prescribed OHA will be designated hereinafter as 'start', 'addition' or 'switch'. Patients will be treated in daily clinical practice and followed up for 3 years.

A total of 20000 patients will be registered and divided into two groups (A and B) consisting of 10000 each, according to the type of OHA that has been started, added on or switched at the time of registration (see figure 2). Group A will include 10000 patients treated with alogliptin, while group B will include 10000 patients not treated with any DPP-4 inhibitor but with other OHA(s). Each participating patient will choose either treatment group according to his or her free will. Approximately 300 medical institutions nationwide will participate in the present study, and each investigational site is expected to enrol eligible patients at a 1:1 ratio as assigned to groups A and B, respectively. See Statistical 
Figure 1 Schematic of study design (OHA, oral hypoglycaemic agent).

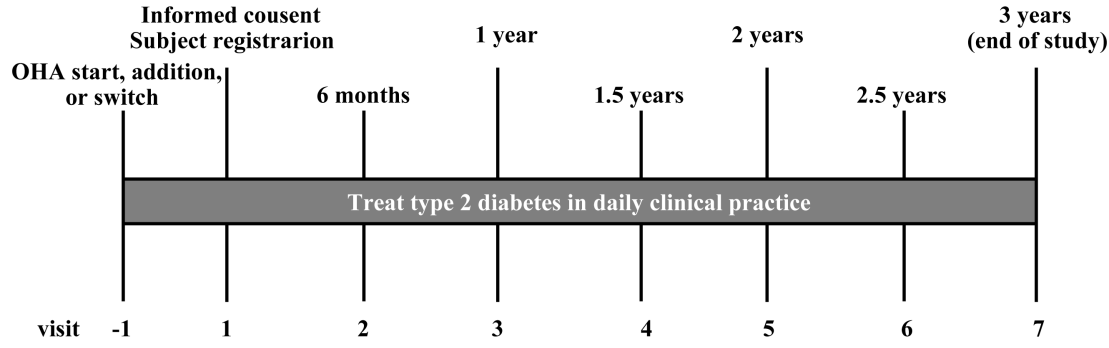

and analytical plans for the rationale for planned sample size.

The study design is schematised in figure 1 .

Each participant's data will be registered every 6 months, totalling 7 times of data registration for a 3-year follow-up period (also see table 1). The 6-month interval of data registration appears reasonable to observe safety and efficacy parameters, e.g. plasma glucose control in participants who will be in daily clinical practice along with diet and exercise therapy after a change in pharmacotherapy (e.g. 'start', 'addition', 'switch' or dose increase or reduction) and to ease the burden on each participant.

\section{Participant eligibility}

Participant eligibility will be determined as summarised in box 1 (also see figure 2 for participant grouping).

Participation of a participant will be discontinued at the discretion of the principal investigator or subinvestigator if any of the following conditions occur: major protocol deviation, lost to follow-up, voluntary withdrawal, study termination, pregnancy or any other reason for which the investigator judges that discontinuation would be necessary.

\section{Treatment}

Treatment with an OHA will be provided in daily clinical practice and may be changed or discontinued within the 3-year observation period, as per the package insert for each OHA. Similarly, non-OHA antidiabetic therapies as well as treatment for concurrent medical conditions will be provided in daily clinical practice, as needed.

\section{Study procedures}

The principal investigator and subinvestigator will observe and assess each participant from the time of informed consent through the completion of observation according to the procedures depicted in table 1 .

All examinations, observations and evaluations should be performed by the principal investigator (or subinvestigator) at the designated time points.
Figure 2 Schematic of participant grouping (DPP-4, dipeptidyl peptidase-4; OHA, oral hypoglycaemic agent).

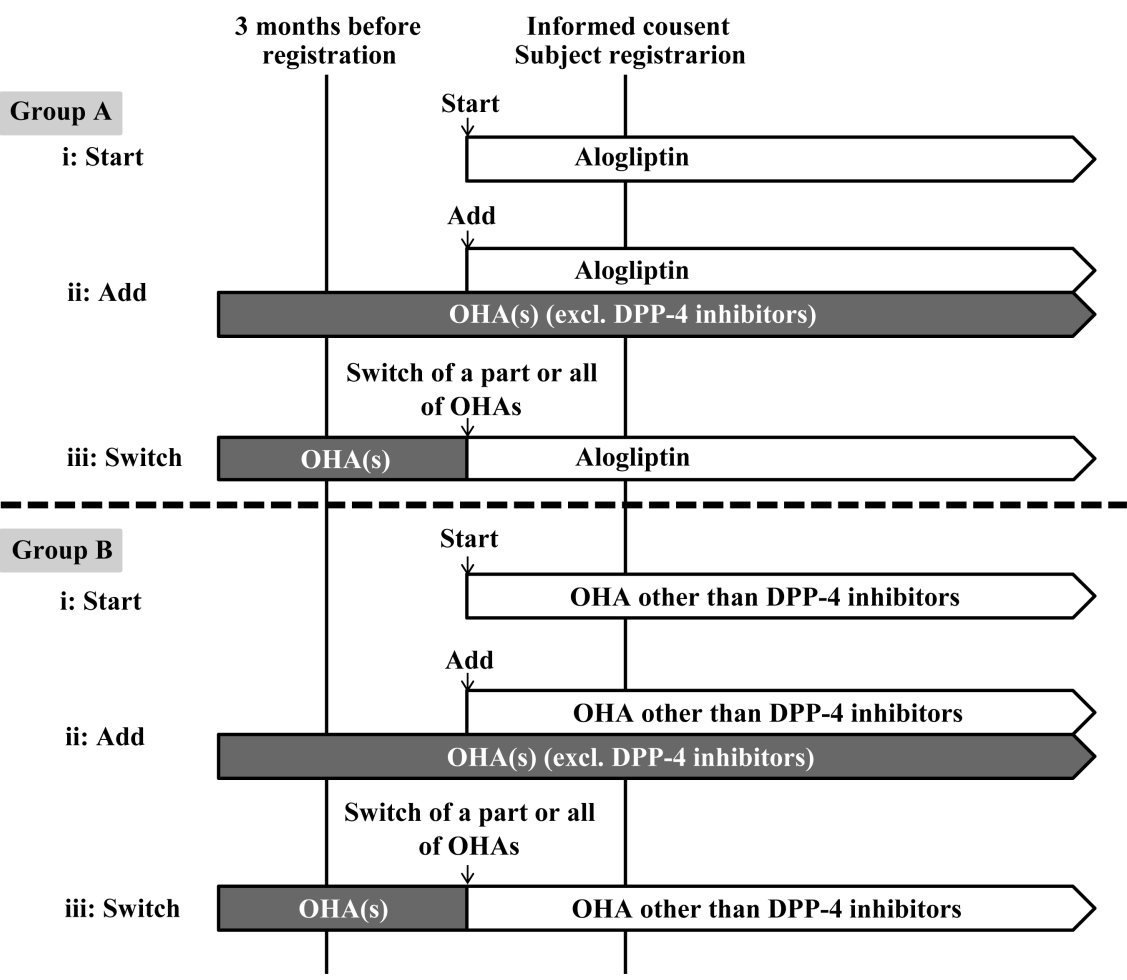


Table 1 Schedule of observations

\begin{tabular}{|c|c|c|c|c|c|c|c|c|c|}
\hline \multirow[b]{3}{*}{ Study month (month) } & \multirow{2}{*}{$\begin{array}{l}\text { Baseline } \\
\text { OHA start, } \\
\text { addition or } \\
\text { switch* }^{*}\end{array}$} & \multirow{3}{*}{$\begin{array}{l}\text { Registration } \\
0\end{array}$} & \multirow{2}{*}{\multicolumn{3}{|c|}{ Observation period }} & \multirow[b]{3}{*}{24} & \multirow[b]{3}{*}{30} & \multirow[b]{3}{*}{36} & \multirow[b]{3}{*}{$\begin{array}{l}\text { Early } \\
\text { termination }\end{array}$} \\
\hline & & & & & & & & & \\
\hline & -3 & & 6 & 12 & 18 & & & & \\
\hline Visit windows (month) & $-3-0$ & 0 & $1-9$ & $10-15$ & $16-21$ & $22-27$ & 28-33 & $34-39$ & - \\
\hline Visit number & -1 & 1 & 2 & 3 & 4 & 5 & 6 & 7 & - \\
\hline Inclusion/exclusion criteria & & $x$ & & & & & & & \\
\hline Informed consent & & $x$ & & & & & & & \\
\hline Demographics & & $x$ & & & & & & & \\
\hline $\begin{array}{l}\text { Medical history/medication } \\
\text { history }(\mathrm{OHA})\end{array}$ & $x$ & & & & & & & & \\
\hline Physical examination & $x$ & $x$ & $x$ & $x$ & $x$ & $x$ & $x$ & $x$ & $x$ \\
\hline Height & $\mathrm{x}$ & & & & & & & & \\
\hline Waist circumference & $\mathrm{x}$ & & $(X)$ & $x$ & $(X)$ & $x$ & $(X)$ & $x$ & $x$ \\
\hline Weight & $\mathrm{x}$ & & $\mathrm{x}$ & $\mathrm{x}$ & $\mathrm{x}$ & $\mathrm{X}$ & $\mathrm{x}$ & $\mathrm{X}$ & $\mathrm{x}$ \\
\hline $\begin{array}{l}\text { Vital signs (sitting blood } \\
\text { pressure and pulse) }\end{array}$ & $x$ & & $x$ & $x$ & $x$ & $x$ & $x$ & $x$ & $x$ \\
\hline Concomitant medications & & & & $x$ & & & & & $x$ \\
\hline Safety end points & $\mathrm{x}$ & & $\mathrm{x}$ & $\mathrm{x}$ & $\mathrm{x}$ & $\mathrm{X}$ & $\mathrm{x}$ & $\mathrm{x}$ & $\mathrm{x}$ \\
\hline Microangiopathy & $\mathrm{x}$ & & $x$ & $x$ & $\mathrm{x}$ & $x$ & $x$ & $x$ & $x$ \\
\hline Macroangiopathy & $\mathrm{x}$ & & & & & & & & \\
\hline Other concurrent conditions & $\mathrm{x}$ & & & & & & & & \\
\hline Clinical laboratory tests $†$ & $x$ & & $x$ & $x$ & $x$ & $x$ & $x$ & $x$ & $x$ \\
\hline HbA1c (NGSP) & $\mathrm{x}$ & & $x$ & $x$ & $\mathrm{x}$ & $x$ & $x$ & $x$ & $x$ \\
\hline Fasting insulin $†$ & $\mathrm{x}$ & & $(X)$ & $x$ & $(X)$ & $x$ & $(X)$ & $x$ & $x$ \\
\hline Fasting blood glucose $\dagger$ & $\mathrm{x}$ & & $(X)$ & $x$ & $(X)$ & $x$ & $(X)$ & $x$ & $x$ \\
\hline Casual blood glucose & $(\mathrm{X})$ & & $(X)$ & $(X)$ & $(X)$ & $(X)$ & $(X)$ & $(X)$ & $(X)$ \\
\hline Fasting and casual $C$ peptide & $(X)$ & & $(X)$ & $(X)$ & $(X)$ & $(X)$ & $(X)$ & $(X)$ & $(X)$ \\
\hline 1,5-AG $\neq$ & $(X)$ & & $(X)$ & $(X)$ & $(X)$ & $(X)$ & $(X)$ & $(X)$ & $(X)$ \\
\hline Glycoalbumin $\ddagger$ & $(X)$ & & $(X)$ & $(X)$ & $(X)$ & $(X)$ & $(X)$ & $(X)$ & $(X)$ \\
\hline Amylaseł & $(X)$ & & $(X)$ & $(X)$ & $(X)$ & $(X)$ & $(X)$ & $(X)$ & $(X)$ \\
\hline Chest X-ray $\ddagger$ & $(\mathrm{X})$ & & $(X)$ & $(X)$ & $(X)$ & $(X)$ & $(X)$ & $(X)$ & $(X)$ \\
\hline 12-lead ECG $\ddagger$ & $(X)$ & & $(X)$ & $(X)$ & $(X)$ & $(X)$ & $(X)$ & $(X)$ & $(X)$ \\
\hline Adverse events & & & & $x$ & & & & 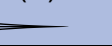 & $x$ \\
\hline
\end{tabular}

$\mathrm{X}$ : examination and observation must be performed.

$(\mathrm{X})$ : examination and observation should be performed, if required.

$\leftarrow X \rightarrow$ : examination and observation must be performed throughout the period.

*The results of examination and observation of essential items and optional items performed on a day closest to the date of visit for the OHA start, addition or switch (within 6 months before registration including the day of registration) should be collected to the extent possible.

†Measurement should be performed in the fasting state (after at least $10 \mathrm{~h}$ of fasting), if possible.

†Optional test items.

AG, Anhydroglucitol; HbA1c, glycated haemoglobin; NGSP, National Glycohemoglobin Standardization Program; OHA, oral hypoglycaemic agent.

\section{Informed consent and participant registration}

The principal investigator (or subinvestigator) will consecutively provide an explanation regarding the study to each eligible participant, using the informed consent document. Participants who give written consent will be registered in the electronic case report form in order, and then observations will be initiated. Participant registration will be closed at each investigational site at the time the target number of participants has been enrolled in alogliptin-treated group (group A) and DPP-4 inhibitor-untreated group (group B). The participant registration for the entire study will also be closed at the time the planned total number of participants for each group (10000 participants) has been reached nationwide. The principal investigator will prepare a list of participant identification numbers and assign a studyspecific, anonymised and uniquely given number to each participant at the time of informed consent to protect the participant's private information. These unique numbers will be used and not changed throughout the study.

\section{Data collection}

Demographics, medical history and medication history

Demographic information to be obtained will include date of birth, gender, height, weight, waist circumference, 


\section{Box 1 Criteria for participant inclusion and exclusion}

\section{Inclusion criteria}

1 Patients who have received a diagnosis of type 2 diabetes and are treated with at least one oral hypoglycaemic agent (OHA) as per the package insert for the OHA

2 Patients who have started or added an $\mathrm{OHA}$, or switched* from one OHA to another within the previous 3 months and meet one of the following criteria (see figure 2):

Group A: Participants treated with alogliptin at the time of registration ('alogliptin-treated group')

I Start: Patients who were with no previous OHA treatment and have newly started alogliptin.

II Addition: Patients who have added alogliptin to $\mathrm{OHA}(\mathrm{s})$ currently taken (excluding dipeptidyl peptidase-4 (DPP-4) inhibitors).

III Switch: Patients who have switched a part or all of OHAs currently taken to alogliptin.

Group B: Participants not treated with any DPP-4 inhibitor at the time of registration ('DPP-4 inhibitor-untreated group')

I Start: Patients who were with no previous OHA treatment and have newly started an OHA other than DPP-4 inhibitors.

II Addition: Patients who have added another OHA, other than DPP-4 inhibitors, to $\mathrm{OHA}(\mathrm{s})$ currently taken (excluding DPP-4 inhibitors).

III Switch: Patients who have switched a part or all of OHAs currently taken to another OHA, other than DPP-4 inhibitors, and are not treated with any DPP-4 inhibitor at the time of registration.

*This applies to active pharmaceutical ingredients only. A switch from one OHA (including combination drug products) to another of the same active pharmaceutical ingredient will not be included in the switch category.

3 Gender and age: men and women of at least 20 years of age at the time of informed consent.

4 Patients willing and able to provide written informed consent prior to participation in this study.

\section{Exclusion criteria}

Any participant who meets any of the following criteria will not qualify for entry into the study:

1 Patients using parenteral hypoglycaemic agents (insulin and glucagon-like peptide-1 receptor agonists).

2 Patients with severe ketosis, diabetic coma or precoma, severe infection, or serious trauma and patients under perioperative management.

3 Pregnant or lactating women.

4 Patients judged by the principal investigator or subinvestigator to be ineligible for participation as study participants for any other reason.

smoking status, drinking habits, family history of cancer and diabetes in first-degree or second-degree relatives, and time of onset or diagnosis of type 2 diabetes.

Medical history will include the presence or absence of the following clinically significant symptoms or diseases that have disappeared or been resolved before the OHA start, addition or switch: severe ketosis (ketoacidosis), diabetic coma or precoma, severe infections, hypoglycaemia (excluding severe hypoglycaemia), severe hypoglycaemia (requiring assistance from others), pancreatitis, cancer (pancreatic cancer and other types), skin disorders, microangiopathy and other clinically significant symptoms or diseases judged by the principal investigator (or subinvestigator).

Ongoing conditions are considered concurrent medical conditions (see the later section of Concurrent medical conditions).

Medication history will include any OHAs stopped within 3 months before the defined OHA start, addition or switch (see figure 2).

\section{Physical examination procedures}

A baseline physical examination will consist of the following body systems: eyes, ears, nose, throat, cardiovascular system, respiratory system, gastrointestinal system, dermatological system, extremities, musculoskeletal system, nervous system, lymph nodes, genitourinary system and others. All physical examinations performed after the OHA start, addition or switch should assess clinically significant changes from the baseline examination.

\section{Major safety end points}

The major safety end points are set in the J-BRAND Registry study as below, and the baseline physical examination will include an assessment of these end points.

- Hypoglycaemia (presence or absence; severity'; symptomatic or asymptomatic; blood glucose level at onset);

- Pancreatitis (presence or absence; type i.e. acute, chronic—chronic or progressive stage—or other);

- Skin disorders (presence or absence; disease name);

- Infections (presence or absence; disease name);

- Cancer (presence or absence; pathogenesis i.e. primary, recurrent, metastatic or unknown; disease name).

\section{Microangiopathy and other measurements}

Microangiopathy will be assessed for new onset or progression of disease. A funduscopic method will be used for the diagnosis of diabetic retinopathy based on Davis classification as simple retinopathy or more advanced. The diagnosis of diabetic neuropathy will be made based on the classification proposed by Toronto Diabetic Neuropathy Expert Group as Probable DPN. ${ }^{18}$ Diabetic nephropathy will be made based on urinary albumin/ creatinine ratio as $30 \mathrm{mg} / \mathrm{g}$ Cr or higher.

Height, waist circumference and weight will be measured in each participant. Body mass index (BMI) will be calculated using metric units with a formula: BMI=weight $(\mathrm{kg}) /$ height $(\mathrm{m})^{2}$. Vital sign measurements will include diastolic and systolic blood pressure $(\mathrm{mm} \mathrm{Hg})$ and pulse $(\mathrm{bpm})$ in a sitting position after a rest of $5 \mathrm{~min}$ or longer.

${ }^{\mathrm{i}}$ Severe hypoglycaemia is defined as hypoglycaemia requiring assistance from others and will be separately collected. 


\section{Concomitant medications}

Detailed information (drug name, duration and daily dose) will be obtained on all OHAs administered during the period from the OHA start, addition or switch to the completion of observation. Information on any medication other than OHAs will be similarly collected for drug name and duration of use.

\section{Concurrent medical conditions}

Concurrent medical conditions are ongoing conditions or diseases that are present at the OHA start, addition or switch. This will include clinically significant abnormalities observed in laboratory tests, ECG or physical examination, as judged by the principal investigator (or subinvestigator). An investigation will be performed for the presence or absence of (1) microangiopathy; (2) macroangiopathy (cerebral infarction, cerebral haemorrhage, myocardial infarction, angina pectoris and foot lesions such as arteriosclerosis obliterans of the lower extremities (Fontaine stages I-IV), foot deformity/callus formation, tinea pedis including tinea unguium, and other infections); and (3) the following conditions.

Other concurrent conditions:

- Lifestyle-related diseases (hypertension, dyslipidaemia and hyperuricaemia);

- Pulmonary disease (interstitial pneumonia);

- Hepatic diseases (fatty liver, alcoholic hepatitis, chronic hepatitis, viral hepatitis and cirrhosis);
- Pancreatic diseases (chronic pancreatitis and acute pancreatitis);

- Renal diseases (nephrotic syndrome, glomerulonephritis and chronic renal failure);

- Cardiac diseases (cardiac failure: New York Heart Association (NYHA) class II, III or IV);

- Allergic diseases (bronchial asthma, pollinosis, allergic rhinitis and allergic dermatitis);

- Autoimmune diseases (rheumatoid arthritis and other autoimmune diseases);

- Cancer (gastric cancer, lung cancer, colorectal cancer, pancreatic cancer, thyroid cancer and other cancers);

- Other symptoms or diseases deemed to be concurrent medical conditions based on the judgement of the principal investigator (or subinvestigator).

\section{Clinical laboratory tests}

Laboratory tests will be performed according to the schedule shown in table 1 . The 'essential items' and 'optional items' are defined for the tests as below (also see table 2) and samples should be collected in the fasting state (after at least $10 \mathrm{~h}$ of fasting), whenever possible. The investigator should collect at-registration (baseline) laboratory data as much as possible on 'essential items' and 'optional items', on a day within 6 months before registration (including the day of registration) and closest to the date of physical examination

Table 2 Essential items and optional items to be collected

\begin{tabular}{|c|c|c|}
\hline Haematology & Blood biochemistry & Urinalysis \\
\hline \multicolumn{3}{|l|}{ Essential items } \\
\hline $\mathrm{HbA1c}$ & Serum creatinine & Urinary albumin \\
\hline Fasting blood glucose* & $\begin{array}{l}\text { Lipid profile (total cholesterol, HDL-C, } \\
\text { LDL-C (calculated), fasting triglycerides) }\end{array}$ & \\
\hline \multirow[t]{2}{*}{ Fasting insulin* } & AST (GOT) & \\
\hline & ALT (GPT) & \\
\hline \multicolumn{3}{|l|}{ Optional items $†$} \\
\hline \multirow{2}{*}{$\begin{array}{l}\text { Red blood cell count } \\
\text { Haemoglobin }\end{array}$} & Total protein & Protein (qualitative) \\
\hline & Blood urea nitrogen & Glucose (qualitative) \\
\hline Haematocrit & Uric acid & Ketone bodies (qualitative) \\
\hline Platelet count & Total bilirubin & Occult blood (qualitative) \\
\hline White cell count & ALP & \\
\hline Differential white blood cells & CK (CPK) & \\
\hline (neutrophils, eosinophils, & $\mathrm{LDH}$ & \\
\hline \multirow{2}{*}{$\begin{array}{l}\text { basophils, lymphocytes and } \\
\text { monocytes) }\end{array}$} & $\gamma$-GTP & \\
\hline & Amylase & \\
\hline \multicolumn{3}{|l|}{ Other } \\
\hline \multicolumn{3}{|l|}{ Casual blood glucose } \\
\hline \multicolumn{3}{|l|}{$1,5-\mathrm{AG}$} \\
\hline \multicolumn{3}{|l|}{ Glycoalbumin } \\
\hline \multicolumn{3}{|l|}{ Fasting $\mathrm{C}$ peptide concentration } \\
\hline \multicolumn{3}{|c|}{ Casual serum $\mathrm{C}$ peptide concentration } \\
\hline \multicolumn{3}{|c|}{$\begin{array}{l}\text { ‘These parameters must be measured at least once a year. } \\
\text { †'Optional items' are the data to be collected if measured. } \\
\text { AG, anhydroglucitol; ALP, alkaline phosphatase; ALT, alanine aminotransferase; AST, aspartate aminotransferase; CK, creatine kinase; } \\
\text { HbA1c, glycated haemoglobin, HDL-C, high-density lipoprotein-cholesterol; LDH, lactate dehydrogenase; LDL-C, low-density } \\
\text { lipoprotein-cholesterol; } \gamma \text {-GTP, } \gamma \text {-glutamyl transpeptidase. }\end{array}$} \\
\hline
\end{tabular}


after the OHA start, addition or switch. Alc (\%) will be collected as NGSP conversion of a conventional HbAlc determination.

\section{Chest X-ray}

Chest radiography will be performed in daily clinical practice, as required. The principal investigator (or subinvestigator) or a radiologist at each investigational site will assess chest X-ray images based on the following categories: normal; abnormal but not clinically significant or abnormal and clinically significant.

\section{Standard 12-lead ECG procedure}

A standard 12-lead ECG will be recorded in daily clinical practice, as required. The principal investigator (or subinvestigator) or a specialist at each investigational site will interpret the ECG based on the following categories: normal; abnormal but not clinically significant; abnormal and clinically significant.

\section{Adverse event collection periods}

Adverse events will be collected during the three collection periods as follows:

- All adverse events occurring between the time of OHA start, addition or switch (Visit -1; see the visit number in table 1) and start of observation (Visit 1);

- All adverse events occurring between the start of observation (Visit 1) and the end of observation (Visit 7);

- Adverse events occurring after completion of observation and are judged to be related to OHA(s).

Any adverse event will be assessed with respect to its name, seriousness, severity, causality to OHA(s) used, date of onset, date of resolution, frequency, action taken with regard to OHAs and outcome.

\section{Statistical and analytical plans}

Three different analysis sets are defined in this study. Full analysis set (FAS) is defined as a group of registered participants who have visited the investigational site at least once after the OHA start, addition or switch. Safety analysis set (SAS) is defined as a group of registered participants who will meet the eligibility criteria (based on the inclusion/exclusion criteria; see box 1) and who have visited the investigational site at least once after the OHA start, addition or switch. Efficacy analysis set (EAS) is defined as a group of registered participants who will meet the eligibility criteria and have visited the investigational site at least once after the registration.

For safety analyses, SAS will be the primary set while FAS will be the secondary. EAS will be used for efficacy analyses. As for new onset or progression of microangiopathy among the efficacy end points, however, analyses will be performed for the SAS population.

Hypoglycaemia, pancreatitis, skin disorders, infections and cancer will be of particular interest for the safety assessment. These outcomes will be analysed primarily by Kaplan-Meier method for estimation of cumulative incidence and by log-rank test for between-group comparison. If necessary, multivariate methods including Cox regression may be used to adjust the baseline differences.

Of efficacy outcomes, A1c, fasting blood glucose, fasting insulin and urinary albumin will be measured at each visit and the change from baseline (at registration) will be compared between groups by two-sided $\mathrm{t}$ test. Missing data will be imputed by last-observation-carriedforward method. If necessary, multivariate methods including analysis of covariance may be used for adjustment of baseline differences. The effect of OHA(s) on the new onset of microangiopathy and its progression will be assessed by Kaplan-Meier method and log-rank test, as performed in hypoglycaemia assessment.

\section{Rationale for planned sample size}

With respect to the sample size described above, approximately 9200 patients will be required to evaluate the safety of alogliptin as the primary end point, detecting at least one adverse event occurring with an incidence of less than $0.05 \%$ with a probability of at least $99 \%$. The same sample size also needs to be set for the DPP-4 inhibitor-untreated group (group B) in order to compare the safety end point. Assuming that 5-7\% of participants are excluded from analysis population, a total of 20000 patients will be required.

Based on the experience in preapproval clinical studies of alogliptin, the incidence of hypoglycaemia is assumed to be 14 events/1000 patient-years in group A and 1.3 times higher in group B than in group A. Assuming that 10000 participants in each group are followed up for 3 years, with a dropout rate of $20 \%$ per annum, the statistical power of log-rank test will be $93.7 \%$ at a two-sided significance level of $5 \%$.

On the basis of a report by Garg et al, ${ }^{19}$ the incidence of acute pancreatitis is assumed to be 5 events/1000 patient-years in group B and 1.5 times higher in group A than in group B. The statistical power of log-rank test under the same conditions as above will be $91.9 \%$ at a significance level of $5 \%$.

Based on the presented rationale, the planned sample size (10 000 participants per group; 20000 in total) will have a sufficient statistical power to meet the objective of this study.

\section{Biological sample use, retention and destruction}

The principal investigator should establish a management system required to protect the participants' personal information and comply with the investigational site's rules regarding sample collection, retention and destruction.

\section{ETHICS AND DISSEMINATION}

This study will be conducted with the highest respect for individual participants according to the protocol, the Declaration of Helsinki, the Ethical Guidelines for Clinical 
Research (Japan Ministry of Health, Labour and Welfare, 2008 Revision), and relevant laws and regulations.

Each study participant will be protected against invasion of privacy. The source data of a participant will be linked throughout the study to the study database or relating documentation(s) via a study-specific, anonymysed and uniquely given number. As permitted by all applicable laws and regulations, limited participant attributes such as gender and date of birth may be used to verify the participant and accuracy of the participant's unique number.

\section{DISCUSSION AND DISSEMINATION}

Based on a search for English language clinical trials previously published, a review article was recently published in which the tolerability of DPP-4 inhibitors was supported on the basis of 5 drugs within this class. ${ }^{9}$ However, in order to provide more comprehensive information with respect to efficacy, safety and tolerability of this class of medications, a contributing database construction needs to be established. The J-BRAND Registry will thus help promote the appropriate use of DPP-4 inhibitors such as alogliptin through long-term (3-year) follow-ups in 10000 DPP-4 inhibitor (alogliptin)receiving patients with type 2 diabetes and another 10000 patients receiving non-DPP-4 inhibitor OHA(s).

The findings of this study will be presented at relevant conferences and published in peer-reviewed journals.

\section{Author affiliations}

${ }^{1}$ Department of Diabetes, Endocrinology and Nutrition, Graduate School of Medicine, Kyoto University, Kyoto, Japan

${ }^{2}$ Department of Diabetes and Metabolic Diseases, Graduate School of Medicine, The University of Tokyo, Tokyo, Japan

${ }^{3}$ Division of Endocrinology, Metabolism, Hematological Sciences and Therapeutics, Yamaguchi University Graduate School of Medicine, Yamaguchi, Japan

${ }^{4}$ Department of Metabolism and Endocrinology, Juntendo University Graduate School of Medicine, Tokyo, Japan

${ }^{5}$ Division of Diabetes, Department of Internal Medicine, Aichi Medical University School of Medicine, Aichi, Japan

${ }^{6}$ Department of Endocrinology, Diabetes and Geriatric Medicine, Akita University Graduate School of Medicine, Akita, Japan

${ }^{7}$ Department of Metabolic Medicine, Graduate School of Medicine, Osaka University, Osaka, Japan

${ }^{8}$ Division of Diabetes, Metabolism and Endocrinology, Department of Internal Medicine, Jikei University School of Medicine, Tokyo, Japan

${ }^{9}$ Graduate School of Public Health, University of Pittsburgh, Pittsburgh, Pennsylvania, USA

${ }^{10}$ Clinical Research Support Center, Graduate School of Medicine, The University of Tokyo, Tokyo, Japan

Acknowledgements At each study site and committee, the investigator is responsible for patient recruitment and performance of all study-related procedures including analysis of the study data, independent of Takeda Pharmaceutical Company Limited.

Contributors NI led the committee to elaborate the study design and study protocol. He was responsible for journal selection and preparation of this article as the principal author. He incorporated coauthors' comments collectively in the article for finalisation. KU, YT and HW elaborated the study design and study protocol and reviewed this article. JN, YY, IS and RN reviewed this article. TY prepared the statistical analysis plan (SAP) for this study. He reviewed this article. TK leads and controls the whole study's activities. He reviewed this article. All authors and coauthors finally approved the manuscript presently submitted.

Funding The J-BRAND Registry is funded by a research grant from Takeda Pharmaceutical Company, Limited.

Competing interests $\mathrm{NI}$ is on the Advisory Board of Takeda, Mitsubishi Tanabe, Nippon Boehringer Ingelheim and Sanofi. He has received honoraria for lectures from MSD, Sanofi, Novartis, Eli Lilly, Kyowa Hakko Kirin, Nippon Boehringer Ingelheim and Mitsubishi Tanabe, and has clinical commissions from MSD, Eli Lilly and Mitsubishi Tanabe. He has research grants supported by MSD, Nippon Boehringer Ingelheim, Sanofi, Daiichi-Sankyo, Takeda, Mitsubishi Tanabe, Novartis, Kyowa Hakko Kirin and AstraZeneca. KU has received honoraria for lectures from Nippon Boehringer Ingelheim, Eli Lilly, MSD, Novartis and Takeda. He has research grants supported by Astellas, Daiichi-Sankyo, MSD, Novartis, Sanofi and Takeda. YT has received honoraria for lectures from MSD, Ono, Sanofi, Dainippon Sumitomo, Takeda, Mitsubishi Tanabe, Nippon Boehringer Ingelheim, Novartis and Novo Nordisk. He has research grants supported by Astellas, MSD, Kyowa Hakko Kirin, Sanofi, Dainippon Sumitomo, Daiichi-Sankyo, Takeda, Nippon Boehringer Ingelheim and Novartis. HW has received honoraria for lectures from Nippon Boehringer Ingelheim, Sanofi, Ono, Novo Nordisk, Novartis, Eli Lilly, Sanwa Kagaku Kenkyusho, Daiichi-Sankyo, Takeda, MSD, Dainippon Sumitomo and Kowa. He has research grants supported by Nippon Boehringer Ingelheim, Pfizer, Mochida, Sanofi, Novo Nordisk, Novartis, Sanwa Kagaku Kenkyusho, Terumo, Eli Lilly, Mitsubishi Tanabe, Daiichi-Sankyo, Takeda, MSD, Shionogi, Dainippon Sumitomo, Kissei and AstraZeneca. JN has received honoraria for lectures from Ono, Pfizer, Novo Nordisk, Eli Lilly, Novartis, Sanofi, MSD and Takeda. He has research grants supported by Ono, Mitsubishi Tanabe, JT, Sanofi, MSD and Takeda. YY is on the Advisory Board of Ono and Novo Nordisk. He has received honoraria for lectures from Takeda, Sanofi and Mitsubishi Tanabe. He has research grants supported by Takeda, Ono and Sanwa Kagaku Kenkyusho. IS is on the Advisory Board of Ono and on the Governing Board of a clinical study Trazenta Randomized study for USing insulin patient of Type 2 Diabetes (TRUST 2 Study) under an agreement with Japan Society for Patient Reported Outcome. He has received honoraria for lectures from Astellas, MSD, Ono, Kowa, Takeda, Mitsubishi Tanabe, Nippon Boehringer Ingelheim and Novartis, and has clinical commissions from Takeda, Eli Lilly and Novartis. He has research grants supported by MSD, Kissei, Kyowa Hakko Kirin, Sanofi, Daiichi-Sankyo, Takeda, Mitsubishi Tanabe, Teijin, Nippon Boehringer Ingelheim, Novartis, Novo Nordisk, Pfizer and Mochida. RN has research grants supported by Astellas, Nippon Boehringer Ingelheim, Novartis, Takeda and Japan Diabetes Foundation. TY has received honoraria for lectures from AstraZeneca, MSD, Kyowa Hakko Kirin, Kowa, Shionogi, Daiichi-Sankyo, Dainippon Sumitomo, Takeda, Novartis and Pfizer, and has clinical commissions from AstraZeneca, MSD, Shionogi and Takeda. He has research grants supported by AstraZeneca, MSD, Kowa, Daiichi-Sankyo, Dainippon Sumitomo, Takeda, Mitsubishi Tanabe and Pfizer. TK is on the Advisory Board of Takeda, Chugai, Sanofi, Mitsubishi Tanabe and AstraZeneca. He has research grants supported by MSD, Ono, Novartis, Takeda, Novo Nordisk A/S, Dainippon Sumitomo, Sanofi and Novo Nordisk Pharma A/S. He is also on the Speakers' Bureau of Takeda, MSD, Sanofi, Eli Lilly, Novartis, Astellas and Dainippon Sumitomo.

Ethics approval Ethics approval was provided by the Institutional Review Board set up in each institutional organisation.

Provenance and peer review Not commissioned; externally reviewed.

Open Access This is an Open Access article distributed in accordance with the Creative Commons Attribution Non Commercial (CC BY-NC 3.0) license, which permits others to distribute, remix, adapt, build upon this work noncommercially, and license their derivative works on different terms, provided the original work is properly cited and the use is non-commercial. See: http:// creativecommons.org/licenses/by-nc/3.0/

\section{REFERENCES}

1. Melmed S, Polonsky KS, Larsen PR, et al. Williams textbook of endocrinology. 12th edn. Philadelphia: Elsevier/Saunders, 2012.

2. International Diabetes Federation. IDF diabetes atlas. 5th edn. Brussels, Belgium: International Diabetes Federation, 2011. 
3. Kahn CR, Weir G, King G, et al. eds. Joslin's diabetes mellitus. 14th edn. Philadelphia: Lippincott Williams \& Wilkins, 2004.

4. Srinivasan BT, Jarvis J, Khunti K, et al. Recent advances in the management of the type 2 diabetes mellitus: a review. Postgrad Med J 2008;84:524-31.

5. Olokoba AB, Obateru OA, Olokoba LB. Type 2 diabetes mellitus: a review of current trends. Oman Med J 2012;27:269-73.

6. Levien TL, Baker DE. New drugs in development for the treatment of diabetes. Diabetes Spectr 2009;22:92-106.

7. Pham DQ, Nogid A, Plakogiannis R. Sitagliptin: a novel agent for the management of type 2 diabetes mellitus. Am J Health Syst Pharm 2008:65:521-31.

8. Moritoh Y, Takeuchi K, Asakawa T, et al. Chronic administration of alogliptin, a novel, potent, and highly selective dipeptidyl peptidase-4 inhibitor, improves glycemic control and beta-cell function in obese diabetic ob/ob mice. Eur J Pharmacol 2008;588:325-32.

9. Richard KR, Shelburne JS, Kirk JK. Tolerability of dipeptidyl peptidase-4 inhibitors: a review. Clin Ther 2011;33:1609-29.

10. Elashoff M, Matveyenko AV, Gier B, et al. Pancreatitis, pancreatic and thyroid cancer with glucagon-like peptide-1-based therapies. Gastroenterology 2011;141:150-6.

11. Amori RE, Lau J, Pittas AG. Efficacy and safety of incretin therapy in type 2 diabetes: systematic review and meta-analysis. JAMA 2007;298:194-206

12. Lankas GR, Leiting B, Roy RS, et al. Dipeptidyl peptidase IV inhibition for the treatment of type 2 diabetes: potential importance of selectivity over dipeptidyl peptidases 8 and 9 . Diabetes 2005;54:2988-94

13. Toussi M, Lamy J-B, Toumelin PL, et al. Using data mining techniques to explore physicians' therapeutic decisions when clinical guidelines do not provide recommendations: methods and example for type 2 diabetes. BMC Med Inform Decis Mak 2009;9:28.

14. Filion KB, Joseph L, Boivin JF, et al. Trends in the prescription of anti-diabetic medications in the United Kingdom: a population-based analysis. Pharmacoepidemiol Drug Saf 2009;18:973-6.

15. Shailendra K. Potent antiatherosclerotic effects of alogliptin in addition to its potent antidiabetic effects. Diabetes Metab Syndr Obes 2012;5:121-3.

16. Noda Y, Miyoshi T, Oe $\mathrm{H}$, et al. Alogliptin ameliorates postprandial lipemia and postprandial endothelial dysfunction in nondiabetic subjects: a preliminary report. Cardiovasc Diabetol 2013;12:8.

17. Ayaori M, Iwakami N, Uto-Kondo $\mathrm{H}$, et al. Dipeptidyl peptidase-4 inhibitors attenuate endothelial function as evaluated by flow-mediated vasodilatation in type 2 diabetic patients. J Am Heart Assoc 2013;2:e003277.

18. Tesfaye S, Boulton AJM, Dyck PJ, et al. Diabetic neuropathies: update on definitions, diagnostic criteria, estimation of severity, and treatments. Diabetes Care 2010;33:2285-93.

19. Garg R, Chen W, Pendergrass M. Acute pancreatitis in type 2 diabetes treated with exenatide or sitagliptin. Diabetes Care 2010;33:2349-54. 